\title{
NATURE OF THE CENTRAL SINGULARITY IN SZEKERES MODELS
}

\author{
PANKAJ S. JOSHI \\ Tata Institute of Fundamental Research \\ Homi Bhabha Road, Bombay 400 005, India \\ E-mail: psj@tifrvax.tifr.res.in \\ ANDRZEJ KRÓLAK \\ Institute of Mathematics, Polish Academy of Sciences \\ Śniadeckich 8, 00-950 Warszawa, Poland \\ E-mail:krolak@impan.impan.gov.pl
}

\begin{abstract}
The occurrence and nature of the central naked singularity in aspherical Szekeres models is investigated here, and the strength of the singularity is discussed. The implications for the cosmic censorship hypothesis are considered.
\end{abstract}

1. Introduction. Several gravitational collapse scenarios exist where the final fate of collapse, ensuing from a regular initial data, is a naked singularity. The important question then is the genericity and stability of such singularities. If the initial data subspace, which gives rise to the naked singularity as the end state of collapse, has zero measure in some suitable sense in the set of all initial data, then one may still be able to reformulate the censorship hypothesis in some way. This could be based on a criterion that naked singularities could form in collapse but they may be non-generic in some sense. An investigation of this nature is important because the assumption of censorship is crucial to most of the important results in black hole physics. In fact, when one considers the gravitational collapse in a generic situation, the very existence of black holes requires this hypothesis.

Towards such a purpose, it would be useful to investigate if the naked singularities still persist when one relaxes the assumption of spherical symmetry. Next question would be regarding the strength of such singularities. It was supposed by Tipler [1], and independently by Królak [2,3] that all generic singularities are of strong curvature type.

1991 Mathematics Subject Classification: Primary 83C75.

Research supported in part by KBN grant 2 P301 05007.

The paper is in final form and no version of it will be published elsewhere. 
Explicit examples of naked strong curvature singularities in Królak's sense $([4,5,6])$ and in Tipler's sense $([7,8,9])$ are known in the Tolman-Bondi-Lemaître (TBL) space-times, which represent spherically symmetric inhomogeneous collapse of dust, and in Vaidya radiation collapse. The TBL space-times are special in that they are spherically symmetric and they have matter in the form of irrotational pressureless dust. We need to know whether naked strong curvature singularities occur in more general space-time situations.

In this contribution we discuss a recent result [10] which shows that naked strong curvature singularities occur in aspherical Szekeres models that do not have any Killing vectors. This shows naked singularities do not arise as a result of spherical symmetry. These models are generalizations of the TBL space-times. It is seen that they admit naked singularities that satisfy both the limiting focusing condition and the strong limiting focusing condition, and hence are strong both in the sense of Królak and Tipler.

2. Central singularity in TBL models. In order to decipher the outcome of continual gravitational collapse of a massive cloud, in terms of either a black hole or a naked singularity, we first consider briefly the TBL model which gives an idea of the basic method used here. The metric in this case is given by

$$
\begin{gathered}
d s^{2}=-d t^{2}+\frac{R^{\prime 2}}{1+f} d r^{2}+R^{2}\left(d \theta^{2}+\sin ^{2} \theta d \phi^{2}\right), \\
T^{i j}=\epsilon \delta_{t}^{i} \delta_{t}^{j}, \quad \epsilon=\epsilon(t, r)=\frac{F^{\prime}}{R^{2} R^{\prime}}, \quad \dot{R}^{2}=\frac{F}{R}+f,
\end{gathered}
$$

where $T^{i j}$ is the stress-energy tensor, $\epsilon$ is the energy density, and $R$ is a function of $t$ and $r$. Here the dot and prime denote partial derivatives with respect to parameters $t$ and $r$ respectively. As we are considering collapse, we require $\dot{R}(t, r)<0$. The quantities $F$ and $f$ are arbitrary functions of $r$ and $4 \pi R^{2}(t, r)$ is the proper area of the mass shells which goes to zero when $R(t, r)=0$. The apparent horizon in the interior dust ball lies at $R=F(r)$.

With the integration of equation for $\dot{R}$ above, we have in all three arbitrary functions of $r$, namely $f(r), F(r)$, and $t_{0}(r)$; where the last one indicates the time along the singularity curve. Using the coordinate freedom left in the choice of scaling of $r(R(0, r)=r)$, the number of arbitrary functions reduces to two and the free functions are now $f$ and $F$. The time $t=t_{0}(r)$ corresponds to the shell-focusing singularity $R=0$. The singularity curve $t=t_{0}(r)$ corresponds to the time when the matter shells meet the physical singularity. The function $f(r)$ classifies the space-time as bound, marginally bound, or unbound, depending on the range of its values which are $f(r)<0, f(r)=0, f(r)>0$, respectively. The function $F(r)$ is interpreted as the weighted mass within the dust ball of coordinate radius $r$. Weak energy condition, $T_{i j} V^{i} V^{j} \geq 0$ is assumed for all non-spacelike vectors $V^{i}$ which implies the density $(\epsilon \geq 0)$ everywhere.

We call the singularity to be central singularity if it occurs at $r=0$. Partial derivatives $R^{\prime}$ and $\dot{R}^{\prime}$ can be written as

$$
\left(\frac{\partial R(t, r)}{\partial r}\right)_{t=\mathrm{const} .}=R^{\prime}=(\eta-\beta) P-\left[\frac{1+\beta-\eta}{\sqrt{\lambda+f}}+\left(\eta-\frac{3}{2} \beta\right) \frac{t}{r}\right] \dot{R},
$$




$$
\left(\frac{\partial R^{\prime}(t, r)}{\partial t}\right)_{r=\mathrm{const} .}=\frac{\beta}{2 r} \dot{R}+\frac{\lambda}{2 r P^{2}}\left[\frac{1+\beta-\eta}{\sqrt{\lambda+f}}+\left(\eta-\frac{3}{2} \beta\right) \frac{t}{r}\right],
$$

where we have used the notation, $R(t, r)=r P(t, r), \eta=r F^{\prime} / F, \beta=r f^{\prime} / f, F(r)=r \lambda(r)$. To focus the discussion, we restrict to functions $f(r)$ and $\lambda(r)$ which are analytic at $r=0$ such that $\lambda(0) \neq 0$.

The tangents $K^{r}=d r / d k$ and $K^{t}=d t / d k$ to the outgoing radial null geodesics, with $k$ as the affine parameter, satisfy

$$
\frac{d K^{t}}{d k}+\frac{\dot{R}^{\prime}}{\sqrt{1+f}}=0, \quad \frac{d t}{d r}=\frac{K^{t}}{K^{r}}=\frac{R^{\prime}}{\sqrt{1+f}}
$$

Now the task is to examine the nature of the singularity at $R=0$. In particular, the problem of nakedness or otherwise of the singularity can be reduced to the existence of real, positive roots of an algebraic equation, constructed out of the free functions $F$ and $f$, and their derivatives [7], which constitute the initial data for the problem. Our purpose is to find whether these geodesics terminate in the past at the central singularity $r=0, t=t_{0}(0)$. The exact nature of this singularity $t=0, r=0$ could be analyzed by the limiting value of $X \equiv t / r$ at $t=0, r=0$. If the geodesics meet the singularity with a definite value of tangent then using l'Hospital rule we get

$$
X_{0}=\lim _{t \rightarrow 0, r \rightarrow 0} \frac{t}{r}=\lim _{t \rightarrow 0, r \rightarrow 0} \frac{d t}{d r}=\lim _{t=0, r=0} \frac{R^{\prime}}{\sqrt{1+f}}
$$

where the notation is, $\lambda_{0}=\lambda(0), \beta_{0}=\beta(0), f_{0}=f(0)$ and $Q=Q(X)=P(X, 0)$. Using the expression for $R^{\prime}$, the above can be written as $V\left(X_{0}\right)=0$, where

$$
V(X) \equiv\left(1-\beta_{0}\right) Q+\left(\frac{\beta_{0}}{\sqrt{\lambda_{0}+f_{0}}}+\left(1-\frac{3}{2} \beta_{0}\right) X\right) \sqrt{\frac{\lambda_{0}}{Q}+f_{0}}-X \sqrt{1+f_{0}}
$$

Hence if the equation $V(X)=0$ has a real positive root, the singularity could be naked. In order to be the end point of null geodesics, at least one real positive value $X=X_{0}$ should satisfy the above equation. Clearly, if no real positive root of the above exists, then the singularity $t=0, r=0$ is not naked. Suppose now $X=X_{0}$ is a simple root to $V(X)=0$. To determine whether $X_{0}$ is realized as a tangent along any outgoing singular geodesics to give a naked singularity, one can integrate the equation of the radial null geodesics in the form $r=r(X)$. It is then seen that there is always at least one null geodesic terminating in the past at the singularity $t=0, r=0$, with $X=X_{0}$. In addition, there would be infinitely many integral curves as well, depending on the values of the parameters involved, that terminate at the singularity in the past. It is thus seen [7] that the existence of a real positive root of the equation $V(X)=0$ is the necessary and sufficient condition for the singularity to be naked. Finally, to determine the curvature strength of the naked singularity at $t=0, r=0$, one may analyze the quantity $k^{2} R_{a b} K^{a} K^{b}$ near the singularity, and it is seen that the strong curvature condition is satisfied in that the above quantity remains finite in the limit of approach to the singularity.

3. Central singularity in Szekeres models. We now discuss the application of such a method to the Szekeres models. The Szekeres space-time [11] is a solution of Einstein's equations representing irrotational dust, $G_{a b}=T_{a b}=\rho u_{a} u_{b}, u_{a} u^{a}=1$, 
( $c=8 \pi G=1)$. The metric has the diagonal form,

$$
d s^{2}=d t^{2}-X^{2} d r^{2}-Y^{2}\left(d x^{2}+d y^{2}\right),
$$

where $(r, x, y)$ are comoving spatial coordinates. The solution is given by (we consider the case $\left.Y^{\prime}=\frac{\partial Y}{\partial r} \neq 0\right)$

$$
Y=\frac{R(t, r)}{P(r, x, y)}, \quad X=\frac{P(r, x, y) Y^{\prime}(t, r)}{\sqrt{1+f(r)}} .
$$

Here $f(r)>-1$, and $P=a(r)\left(x^{2}+y^{2}\right)+2 b_{1}(r) x+2 b_{2}(r) y+c(r)$, with $a c-b_{1}^{2}-b_{2}^{2}=1 / 4$. We get $\dot{R}^{2}=f+F(r) / R$, where $F(r)$ is an arbitrary function of $r$, and the dot denotes partial derivative with respect to the time coordinate $t$.

The regularity conditions assumed are, the metric is everywhere $C^{1}$, locally Euclidian at $r=0$ (so $f(0)=0$ ), and $R_{o}(r)=R(r, 0)$ be a monotonically increasing function of $r$. Then $P$ must be everywhere non-zero, and its derivative with respect to $r$ must be continuous and vanishing at $r=0$. We can then use the freedom in the choice of the radial coordinate $r$ to obtain $R_{o}(r)=r$ on the initial time slice. The dust density $\rho$ is given by

$$
\rho=\frac{P F^{\prime}-3 F P^{\prime}}{P^{2} R^{2} Y^{\prime}} .
$$

Although for $P>0$ the surfaces $r=$ const, $t=$ const are spheres, the solution is not spherically symmetric. This is because the spheres are not concentric, their centers being given by $\left(-a^{-1} b_{1},-a^{-1} b_{2}\right)$. Szekeres analyzed the singularities, and their causal structure in his space-times. When $R=0$, the singularity is of the first kind, and when $Y^{\prime}=0$ the singularity is of the second kind. The singularities of the second kind are familiar shellcrossing singularities that also occur in TBL space-times [12]. Like in TBL space-times, shell-crossing singularities in Szekeres spaces can also be both locally and globally naked [11]. However, they are generally believed to be mild and we shall not consider them here. We eliminate these singularities by imposing a regularity condition $Y^{\prime}>0$. Szekeres has also shown that whenever $r>0$, the shell of dust always crosses the apparent horizon before collapsing to singularity. Therefore, for $r>0$, the singularity cannot be naked. It follows that the singularity of the first kind can be naked only when $r=0$, which is the central singularity. This is analogous to the TBL case. We see that like in TBL space-times, the strong curvature naked singularities do occur in Szekeres space-times. For the case of gravitational collapse, we have $\dot{R}<0$. For simplicity, we consider the case of marginally bound collapse, that is, $f(r)=0$. Then the function $R(r, t)$ is given by

$$
R=r\left(1-\frac{3}{2} \sqrt{\frac{F}{r^{3}}} t\right)^{2 / 3}
$$

Following an analysis similar to the TBL case discussed above, the structure of the central singularity can now be analyzed [10]. If the outgoing null geodesics are to terminate in the past at the central singularity $r=0$ at time $t=t_{o}$, when $R\left(t_{o}, 0\right)=0$, then along such trajectories we have $R \rightarrow 0$ as $r \rightarrow 0$. The following is satisfied along null geodesics:

$$
\frac{d R}{d u}=\left(1-\sqrt{\frac{\Lambda}{X}}\right) \frac{H(X, u)}{\alpha}+\frac{\sqrt{X \Lambda}}{\alpha} \mathcal{L} \equiv U(X, u),
$$


where $u=r^{\alpha}, X=R / r^{\alpha}, \eta=r F^{\prime} / F, \Lambda=F / r^{\alpha}, \Theta=(1-\eta / 3) / r^{3(\alpha-1) / 2}, \mathcal{L}=r P^{\prime} / P$ and $H=\frac{\eta X}{3}+\frac{\Theta}{\sqrt{X}}$. The unique value of the constant $\alpha \geq 1$ is determined by the condition that $\Theta / \sqrt{X}$ does not vanish, or go to infinity identically, as $r \rightarrow 0$ in the limit of approach to the central singularity along any $X=$ const. direction. We assume the above functions are at least $C^{2}$.

Let us consider the limit $X_{o}$ of the function $X$ along the null geodesics terminating at the singularity at $R=0, u=0$. Using the l'Hospital rule we get

$$
X_{o}=\lim _{R \rightarrow 0, u \rightarrow 0} \frac{R}{u}=\lim _{R \rightarrow 0, u \rightarrow 0} \frac{d R}{d u}=\lim _{R \rightarrow 0, u \rightarrow 0} U(X, u)=U\left(X_{o}, 0\right)
$$

The necessary condition for the existence of a null geodesic outgoing from the central singularity is the existence of a positive real root $X_{o}$ of the equation $V(X) \equiv U(X, 0)-$ $X=0$. By our regularity conditions we have that $\lim _{r \rightarrow 0} \mathcal{L}=0$. Consequently, the necessary condition for the existence of the naked singularity in the marginally bound case of Szekeres space-times is the existence of real positive roots of the equation

$$
\left(1+\sqrt{\frac{\Lambda_{o}}{X}}\right) \frac{H(X, 0)}{\alpha}-X=0,
$$

where we have put $\eta_{o}=\eta(0), \Lambda_{o}=\Lambda(0), \Theta_{o}=\Theta(0)$. This is the same equation as in the marginally bound TBL case [7]. Hence, the same analysis as in the TBL case applies here. We shall only summarize here the results. To show that the singularity is naked we still need to prove that there exists a solution of the geodesic equations such that the tangent $X_{o}$ is realized at the singularity. One can prove that there is always at least a single null geodesic outgoing from the central singularity ([7] p.5363). Thus, the existence of a real and positive root of the equation above is both necessary and sufficient condition for the existence of a naked singularity. Finally, the strength of this singularity can be investigated, and it is seen that the central singularity considered here is strong, both in the sense of Królak as well as Tipler [10].

The assumption of vanishing pressures here, which could be important in the final stages of the collapse, may be considered as the limitation of dust models. On the other hand, it is also sometimes argued that in the final stages of collapse, the dust equation of state could be relevant as at higher and higher densities the matter may behave more and more like dust. Further, if there are no large negative pressures (as implied by the validity of the energy conditions), then the pressure also might contribute gravitationally in a positive manner to the effect of dust, and may not alter the conclusions.

\section{References}

[1] F. J. Tipler, 1977 Phys. Lett. 67A 8.

[2] A. Królak, 1978 MSc Thesis, Singularities and Black Holes in General Space-times, University of Warsaw, unpublished.

[3] A. Królak, 1986 Class. Quantum Grav. 3267.

[4] D. M. Eardley and L. Smarr, 1979 Phys. Rev. D 192239.

[5] D. Christodoulou, 1984 Commun. Math. Phys. 93171. 
[6] R. P. A. C. Newman, 1986 Class. Quantum Grav. 3527.

[7] P. S. Joshi and I. H. Dwivedi, 1993 Phys. Rev. D 475357.

[8] I. H. Dwivedi and P. S. Joshi, 1989 Class. Quantum Grav. 61599.

[9] I. H. Dwivedi and P. S. Joshi, 1991 Class. Quantum Grav. 81339.

[10] P. S. Joshi and A. Królak, 1996 gr-qc/9605033, To appear in Class. Quantum Grav.

[11] P. Szekeres, 1975 Phys. Rev. D12 2941.

[12] P. Yodzis, H. J. Seifert and H. Muller zum Hagen, 1973 Commun. Math. Phys. 34 135. 